Jochen Taupitz

\title{
7. Organoide: Die deutsche Rechtslage
}

\subsection{Einleitung}

Spezielle Rechtsregeln zur Herstellung und Verwendung von Organoiden existieren in Deutschland nicht. Das bedeutet allerdings nicht, dass sie vom Recht nicht erfasst würden. Einen rechtsfreien Raum gibt es in Deutschland nicht. Vielmehr sind stets mehr oder weniger allgemeine Regeln anwendbar, seien sie auf verfassungsrechtlicher Ebene im Grundgesetz (GG) oder aber im „einfachen“, also unterhalb des Grundgesetzes stehenden Recht, verankert.

Die juristische Einordnung von Organoiden hat wie diejenige anderer Zellen, Gewebe und Organe mehrere Regelungsbereiche in den Blick zu nehmen: Zum einen geht es um die Herkunft bzw. die Gewinnung des Ausgangsmaterials, sodann um die Einordnung der Organoide selbst und schließlich um die Art und Weise ihrer (geplanten) Verwendung. Allerdings stehen die damit angesprochenen Fragen nicht unverbunden nebeneinander. Vielmehr hat insbesondere die geplante Verwendung erheblichen Einfluss bereits auf die Anforderungen, die an die Gewinnung des Ausgangsmaterials zu stellen sind. Auch die rechtliche Einordnung der hergestellten Organoide kann von ihrer geplanten Verwendung beeinflusst sein. Gleichwohl soll der folgende Beitrag die verschiedenen Schritte der Herstellung und Verwendung von Organoiden grundsätzlich getrennt betrachten.

Um den Rahmen des Beitrags nicht zu sprengen, sei bezüglich der verfassungsrechtlichen Rahmenbedingungen lediglich auf die Forschungsfreiheit (Art. 5 Abs. 3 GG) verwiesen, ${ }^{1}$ darüber hinaus auf das Allgemeine Persönlichkeitsrecht (Art. 2 Abs. 1 in Verbindung mit Art. 1 Abs. 1 GG) und das Recht auf Leben und körperliche Unversehrtheit (Art. 2 Abs. 2 GG). Die Letztgenannten und das Allgemeine Persönlichkeitsrecht stellen Abwehrrechte, hier vor allem der Spender der für die Organoide verwendeten Zellen, dar. Aus ihnen folgt bezogen auf (zukünftige) von Organoiden gesundheitlich (eventuell) profitierende Patienten aber auch, dass der Staat nicht ohne hinreichenden

1 Sie ist „vorbehaltlos “ gewährleistet, d. h. in sie darf nur zum Schutz anderer mit Verfassungsrang ausgestatteter Rechtswerte eingegriffen werden. 
Grund z. B. therapeutische Maßnahmen verbieten darf. Schließlich ist auch der Tierschutz als Rechtsprinzip in der Verfassung verankert (Art. 20a GG).

\subsection{Herkunft des Ausgangsmaterials}

\subsubsection{Stammzellgesetz}

Organoide können aus Stammzellen hergestellt werden. Sofern es sich dabei um embryonale Stammzellen handelt, ${ }^{2}$ regelt das Stammzellgeset ${ }^{3}$ die Zulässigkeit ihres Imports nach Deutschland und die Zulässigkeit ihrer inländischen Verwendung. Ihre Gewinnung aus Embryonen ist in Deutschland dagegen nach § 2 Abs. 1 Embryonenschutzgesetz (ESchG) ${ }^{4}$ verboten.

\subsubsection{Allgemeine medizinrechtliche Grundsätze}

Werden dagegen sonstige menschliche Zellen zur Herstellung verwendet (ggf. auch über eine zwischenzeitliche Reprogrammierung der Zellen zu induzierten pluripotenten Stammzellen) ${ }^{5}$ und ist zur Gewinnung der Zellen ein Eingriff in die körperliche Integrität eines Menschen (nachfolgend: eines Spenders) erforderlich, ist hierzu nach allgemeinen Grundsätzen dessen Einwilligung in die mit der Entnahme einhergehenden Körperverletzung notwendig. ${ }^{6}$ Die Wirksamkeit der Einwilligung hängt von einer hinreichenden Aufklärung ab, die insbesondere den Zweck der Entnahme und die mit der Entnahme einhergehenden Risiken umfassen muss. ${ }^{7}$

\subsubsection{Allgemeines Persönlichkeitsrecht des Spenders}

Sofern auf bereits vom menschlichen Körper getrennte Zellen zur Erzeugung der Organoide zugegriffen werden soll, ist das Allgemeine Persönlichkeitsrecht des Spenders

2 Siehe dazu Boers et al., 2016: 938.

3 Stammzellgesetz vom 28. Juni 2002 (BGBl. I: 2277), zuletzt geändert durch Artikel 50 des Gesetzes vom 29. März 2017 (BGBl. I: 626).

4 Embryonenschutzgesetz vom 13. Dezember 1990 (BGBl. I: 2746), zuletzt geändert durch Artikel 1 des Gesetzes vom 21. November 2011 (BGBl. I: 2228).

5 Dazu Faltus, 2016: 289 f.; ausführlich Gerke et al., 2020.

6 Siehe statt vieler BGHZ 29, 46, 49; Schaub, 2018: § 823 Rdnr. 204 mit weiteren Nachweisen. Bei Verwendung fetaler Zellen ist der Informed Consent der Mutter notwendig; vgl. dazu auch § 4a TPG.

7 Siehe z. B. Schröder/Taupitz, 1991: $23 \mathrm{ff}$.; allgemein BGHZ 29, 176, 180; Schaub, 2018 : §823 Rdnr. 207 mit weiteren Nachweisen. 
einschlägig. Denn nach vorherrschender Ansicht setzt sich das Allgemeine Persönlichkeitsrecht des Spenders an den von seinem Körper getrennten Zellen fort, und zwar schon deshalb, weil und soweit darin genetisches Material des Spenders enthalten ist. $^{8}$ Das gilt unabhängig davon, ob an dem Material auch Eigentum (des Spenders oder einer anderen Person, etwa des Forschers) besteht. ${ }^{9}$ Denn beide Rechte können nebeneinander an derselben Sache bestehen.

Das Allgemeine Persönlichkeitsrecht ist als sonstiges Recht im Sinne von § 823 Abs. 1 BGB anerkannt und garantiert nach gefestigter Rechtsprechung des Bundesverfassungsgerichts (BVerfG) $)^{10}$ und des Bundesgerichtshofs $(\mathrm{BGH})^{11}$ gegenüber dem Staat und Dritten die Achtung der Privatsphäre; es gesteht jedermann einen autonomen Bereich der eigenen Lebensgestaltung zu, in dem er seine Individualität unter Ausschluss anderer entwickeln und wahrnehmen kann. ${ }^{12}$ Es wird von den Gerichten aus einem Schutzauftrag aus Art. 1 Abs. 1 und Art. 2 Abs. 1 GG abgeleitet, ${ }^{13}$ weist danach also einen engen Bezug zur Menschenwürde auf. Aus ihm folgt ein Bestimmungsrecht des Spenders darüber, wer was mit „seinen“ Körpermaterialien machen darf. Aufgrund seines Persönlichkeitsrechts hat damit jedermann das Recht, eine Nutzung „seines“ Körpermaterials explizit zu erlauben oder zu verbieten.

Umstritten ist allerdings die Rechtslage, wenn der Spender keine eigene Entscheidung getroffen hat, etwa weil er auf die in Frage stehende Verwendung, hier also die Verwendung der Zellen zur Herstellung von Organoiden, nicht hingewiesen wurde:

a) Nach einer Auffassung ist eine informierte Einwilligung des Patienten zu jeglicher Weiterverwendung seines Körpermaterials notwendig. ${ }^{14}$ Unklar bleibt dabei aber nicht selten, ob das Erfordernis einer entsprechenden Aufklärung und Einwilligung des Patienten lediglich ethisch wünschenswert oder auch rechtlich zwingend ist. ${ }^{15}$ Zum Teil wird in entsprechenden Stellungnahmen ausdrücklich offen gelassen, ob eine

8 Siehe statt vieler Taupitz, 1991: 201, 208 ff.; Schröder/Taupitz, 1991: 35 ff.; Halasz, 2004: 20 ff.; Wicklein, 2007: 82 f., 93 ff.; Roth, 2009: 57 ff.; Breithaupt, 2012: 120 ff., 131 ff.; Wernscheid, 2012: 164 ff.; Taupitz/Schreiber, 2016: 305.

9 Siehe die Nachweise in der vorigen $\mathrm{Fn}$.

10 Siehe etwa BVerfGE 34, 269, 271; 35, 202, 226.

11 Siehe etwa BGHZ 30, 7; 50, 133.

12 Prütting, 2018: § 12 Rdnr. 31.

13 Siehe die Nachweise bei Prütting, 2018: §12 Rdnr. 31.

14 v. Freier, 2005: 321 ff.; Söns, 2008: 245 ff.; Büchner, 2010: 121, 242; Wernscheid, 2012: 168 ff.; siehe auch Deutscher Ethikrat, 2010: $39 \mathrm{ff}$., 55 (aber vor allem mit Blick auf eine zu schaffende gesetzliche Regelung); Nationaler Ethikrat, 2004: 16, 65.

15 Siehe insbesondere die Empfehlungen des Nationalen Ethikrats, 2004: 16, 65; Deutscher Ethikrat, 2010: 42 . 
Einwilligung des Betroffenen aus rechtlicher Sicht erforderlich ist. ${ }^{16}$ Unklar ist auch, ob im Falle einer fehlenden tatsächlichen Einwilligung (sofern sie auch nicht nachträglich eingeholt werden kann) auch eine mutmaßliche Einwilligung ausreichen kann ${ }^{17}$ was zu bejahen ist. Denn wenn eine mutmaßliche Einwilligung schon einen körperlichen Eingriff rechtfertigen kann, vgl. § 630 d Abs. 1 S. 4 BGB, dann muss dies erst recht für eine Verwendung von Körpermaterial gelten, bei der keinerlei Risiken für Körper und Gesundheit des Patienten drohen.

b) Die Auffassung, wonach für jede Art der Weiterverwendung von menschlichen Körpersubstanzen von Rechts wegen eine Einwilligung des ursprünglichen Substanzträgers erforderlich ist, ist nicht überzeugend. Sie widerspricht dem Charakter des Allgemeinen Persönlichkeitsrechts als Rahmenrecht, ${ }^{18}$ bei dem jeweils im Einzelfall zu prüfen ist, ob in der konkreten Nutzung des Körpermaterials eine rechtswidrige Verletzung des Allgemeinen Persönlichkeitsrechts zu sehen ist. ${ }^{19}$ Dabei ist eine umfassende Güter- und Interessenabwägung vorzunehmen, in die sowohl die Belange des Spenders als auch diejenigen des Verwenders des Körpermaterials einzubeziehen sind. ${ }^{20}$ Etwas anderes gilt lediglich dann, wenn der Spender bestimmte Nutzungen ausdrücklich verboten hat. ${ }^{21}$

Für die im Falle des Fehlens einer ausdrücklichen Erklärung des Spenders vorzunehmende Abwägung gilt Folgendes:

Auf Seiten des Verwenders können die allgemeine Handlungsfreiheit (Art. 2 GG), die Forschungsfreiheit (Art. 5 Abs. $3 \mathrm{GG}$ ), die Berufsfreiheit (Art. $12 \mathrm{GG}$ ) und ggf. das Eigentumsrecht (Art. $14 \mathrm{GG}$ ) in die Waagschale geworfen werden.

16 Zentrale Ethikkommission bei der Bundesärztekammer, 2003.

17 Taupitz/Schreiber, 2016: 304, 306.

18 Dazu und zu der Konsequenz, dass die Feststellung eines Eingriffs in das Persönlichkeitsrecht für sich genommen nicht ausreicht, um die Rechtswidrigkeit der Handlung zu bejahen, sondern vielmehr eine Güter- und Interessenabwägung notwendig ist, siehe BGHZ 13, 334 (338); 31, 308 (312 f.); 36, 77 (82 f.); 50, 133 (143); Deutsch, 1992: 163; Prütting, 2018: § 12 Rdnr. 31; Sprau, 2020: § 823 Rdnr. 95 mit weiteren Nachweisen.

19 Siehe statt vieler Taupitz, 1991: 209 ff.; Nationaler Ethikrat, 2004: 52; Wicklein, 2007: 106 ff., 188 ff.; Breithaupt, 2012: 242 ff.; Taupitz/Schreiber, 2016: 304, 305; Schreiber, 2019: 306 ff. mit zahlreichen weiteren Nachweisen.

20 Siehe die Nachweise in der vorigen Fn.; zur internationalen Diskussion Boers et al., 2016: 939.

21 Siehe statt vieler Taupitz, 1991: 211; Nationaler Ethikrat, 2004: 51, 56; Simon et al., 2006: 128; Schreiber, 2019: 311. Siehe auch Ohly, 2003: 426, der dem Betroffenen ohnehin lediglich ein Widerspruchsrecht gegen die Weiterverwendung einräumt. Natürlich führt auch umgekehrt eine ausdrückliche Einwilligung zur Rechtfertigung eines an sich - aufgrund Güterabwägung - rechtswidrigen Eingriffs. 
Auf Seiten des Spenders sind aus dem Blickwinkel des Allgemeinen Persönlichkeitsrechts (Art. 2 i.V.m. Art. 1 GG) neben der Art der Verwendung, der Begleitumstände und dem Ziel der Verwendung (z. B. wären Versuche des Klonierens des Spenders unstreitig rechtswidrig) sowie der Menge des verwendeten Materials ${ }^{22}$ auch weitere Konsequenzen wie insbesondere Gefährdungen für den Spender (auch aus dem Blickwinkel, ob das Material für ihn selbst noch von Nutzen wäre ${ }^{23}$ und vor diesem Hintergrund nicht zuletzt das Ausmaß der Anonymisierung entscheidend. ${ }^{24}$ Denn wenn das Material anonymisiert verwendet wird oder so pseudonymisiert, ${ }^{25}$ dass der Verwender keine Rückschlüsse auf den individuellen Spender ziehen kann (weil er selbst nicht über den Schlüssel zur Identifizierung des Spenders verfügt), kann die Verwendung vereinfacht ausgedrückt - dem Spender nicht schaden. ${ }^{26}$ Insbesondere können Informationen über ihn dann auch nicht von Unberechtigten zum Nachteil des Spenders verwendet werden.

Insgesamt sind persönlichkeitsrechtliche Belange des Spenders vor allem in folgenden drei Fallgruppen tangiert: ${ }^{27}$

- Wenn personenbezogene Daten durch Untersuchung des Körpermaterials gewonnen werden,

- wenn das Körpermaterial mit seinen (und womöglich gerade wegen seiner) individuellen Eigenschaften in andere Personen oder deren Zellen übertragen wird oder wenn es wegen seiner Individualität physisch perpetuiert bzw. vervielfältigt wird,

- wenn es Verwendungen zugeführt wird, über deren rechtliche oder ethische Zulässigkeit oder Vertretbarkeit unter Forschern bzw. in der Öffentlichkeit keine Übereinstimmung herrscht.

22 Taupitz, 2017: 356.

23 Siehe Schreiber, 2019: 308.

24 Siehe die Nachweise in Fn. 19 sowie vor allem Schreiber, 2019: $306 \mathrm{ff}$. mit zahlreichen Nachweisen.

25 Siehe Art. 4 Nr. 5 Datenschutzgrundverordnung (DSGVO): Demnach ist Pseudonymisierung die Verarbeitung personenbezogener Daten in einer Weise, dass die personenbezogenen Daten ohne Hinzuziehung zusätzlicher Informationen nicht mehr einer spezifisch betroffenen Person zugeordnet werden können, sofern diese zusätzlichen Informationen gesondert aufbewahrt werden und technischen und organisatorischen Maßnahmen unterliegen, die gewährleisten, dass die personenbezogenen Daten nicht einer identifizierten oder identifizierbaren natürlichen Person zugewiesen werden.

26 Taupitz, 2017: $356 \mathrm{f}$. Vielfach wird sogar angenommen, dass durch eine Anonymisierung jede persönlichkeitsrechtliche Beziehung erloschen sei, sodass eine Verletzung des allgemeinen Persönlichkeitsrechts von vornherein ausschiede, siehe Fink, 2005: 56 ff.; Antonov, 2006: 107; Breithaupt, 2012: $260,262$.

27 Schröder/Taupitz, 1991: 69; Schreiber, 2019: 308 ff. mit weiteren Nachweisen. 
Abhängig von den Einsatzgebieten der Organoide ist somit eine darauf bezogene Einwilligung des entsprechenden Spenders notwendig (siehe für eine datenschutzrechtliche Einordung Molnár-Gábor, Kap. 8).

Unabhängig von dem vorstehend wiedergegebenen Streit ist bedeutsam, dass die Einholung einer expliziten Einwilligung des Spenders in der Praxis zum Standard im Sinne einer Good Scientific Practice geworden ist. Diese zunehmende Üblichkeit beeinflusst schleichend auch die rechtlichen Anforderungen. ${ }^{28}$ Jedenfalls Körpermaterialien, die in neuerer Zeit gewonnen wurden, sollten daher nur mit einer explizit auf Forschung bezogenen Einwilligung zur Herstellung von Organoiden verwendet werden. Dabei reicht nach zutreffender, aber nicht unumstrittener Auffassung ${ }^{29}$ ein „Broad“ Consent (nicht aber ein „Global“ Consent) aus, der nicht das jeweils einzelne Forschungsprojekt benennt, sondern breiter die Verwendung der Körpermaterialien für „biomedizinische“ Forschung umfasst (siehe zu Formen der Einwilligung auch Molnár-Gábor, Kap. 8). ${ }^{30}$

Über die spezifischen Eigenschaften der (beabsichtigten) Organoide ist dann aufzuklären, wenn es sich um ethisch und rechtlich problematische Erzeugnisse handelt, z. B. Embryoide oder weit entwickelte Hirnorganoide (dazu unten 7.3.1). Über die geplante Verwendung von Organioden ist auch dann aufzuklären, wenn diese im Rahmen von Heilversuchen (oder später einmal im Rahmen klinischer Routine) auf andere Menschen übertragen werden sollen. In anderen Fällen reicht es aus, darüber zu informieren, dass aus den Zellen des Spenders Zellstrukturen hergestellt werden sollen.

\subsubsection{Transplantationsgesetz}

Sofern die späteren Organoide für den klinischen Einsatz bestimmt sind, also auf andere Menschen übertragen werden sollen, kann für die Spende und die Entnahme der zur Herstellung benötigten somatischen Zellen oder Gewebe das Transplantationsgesetz (TPG) ${ }^{31}$ einschlägig sein. Gewebe im Sinne des Gesetzes sind gemäß § 1a Nr. 4 TPG „alle aus Zellen bestehenden Bestandteile des menschlichen Körpers, die keine Organe nach Nummer 1 sind, einschließlich einzelner menschlicher Zellen“. Der Gesetzgeber

28 Taupitz, 2009: $66 \mathrm{ff}$.

29 Nach der Gegenauffassung ist ein Broad Consent nicht „informiert“ genug.

30 Taupitz/Schreiber, 2016: 307; Schreiber, 2019: 294 f. mit weiteren Nachweisen.

31 Transplantationsgesetz in der Fassung der Bekanntmachung vom 4. September 2007 (BGBl. I: 2206), zuletzt geändert durch Artikel 24 des Gesetzes vom 20. November 2019 (BGBl. I: 1626). 
hat sich bewusst für einen weiten Gewebebegriff entschieden, der auch einzelne menschliche Zellen miterfasst. ${ }^{32}$

Das Gesetz „gilt für die Spende und die Entnahme von menschlichen Organen oder Geweben zum Zwecke der Übertragung“ (§ 1 Abs. 2 S. 1 TPG). Die Übertragung ihrerseits ist definiert in § 1a Nr. 7 TPG als die „Verwendung von Organen oder Geweben in oder an einem menschlichen Empfänger sowie die Anwendung beim Menschen außerhalb des Körpers“.

Legt man das Tatbestandsmerkmal „zum Zwecke der Übertragung“ eng aus, dann müssten die menschlichen Organe oder Gewebe, so wie sie entnommen werden, im Zeitpunkt der Entnahme zur Verwendung beim Empfänger bestimmt sein. Hiernach würde das TPG nicht für die Spende und die Entnahme von somatischen Zellen als Ausgangsmaterial zur Herstellung von Organoiden gelten, da nicht die zu entnehmenden Zellen, sondern erst die Produkte im oder am menschlichen Körper angewendet werden sollen.

Geht man dagegen von einem weiten Verständnis aus, so ist auch ein mittelbarer Übertragungszweck im Zeitpunkt der Entnahme erfasst. ${ }^{33}$ Das heißt, dass auch solche im Zeitpunkt der Entnahme zur Weiterverarbeitung bestimmte menschliche Zellen dem Anwendungsbereich des TPG unterliegen, die (nach ihrer Weiterverarbeitung) zur „Verwendung [...] in oder an einem menschlichen Empfänger“ oder zur „Anwendung beim Menschen außerhalb des Körpers“ bestimmt sind. ${ }^{34}$ Dies ist für die Ausgangszellen von Organoiden, die ihrerseits klinisch eingesetzt werden sollen, grundsätzlich der Fall.

Für die weite Auslegung des Tatbestandsmerkmals „zum Zwecke der Übertragung“ spricht, dass bereits der Wortlaut keine Unmittelbarkeit verlangt. Zudem ergibt sich aus der Gesetzesbegründung, dass der Gesetzgeber auch „zur Weiterverarbeitung bestimmte Gewebe, die zunächst be- oder verarbeitet werden, bevor sie bei Menschen verwendet werden“ vom Anwendungsbereich des TPG erfasst wissen wollte. ${ }^{35}$ Darüber

32 BT-Drucks. 16/3146, 2006: 24; Wernscheid, 2012: 111 f.; der Verzicht auf eine eigene Begriffsbestimmung für Zellen lag insbesondere darin begründet, dass die Gewebe-RL die gleichen Anforderungen an Zellen wie an Gewebe stellt.

33 So z. B. Wernscheid, 2012: 71 f. m.w.N. (zum vor der 12. Arzneimittelgesetz-Novelle geltenden TPG).

34 Erfasst ist sowohl die unmittelbare Gewinnung durch Eingriff im oder am menschlichen Körper als auch die mittelbare extrakorporale Gewinnung (z. B. im Fall von Sektions- und Operationsmaterial), siehe BT-Drucks. 16/3146: 21; Gerke et al., 2020b: 292.

35 BT-Drucks. 16/3146: 21. 
hinaus spricht auch das Ziel des (dem TPG zugrundeliegenden) Gewebegesetzes, ${ }^{36}$ die Qualität und Sicherheit von Geweben zu gewährleisten, ${ }^{37}$ für eine weite Auslegung.

Somit ist festzuhalten, dass die Spende und die Entnahme von menschlichen Geweben (einschließlich einzelner Zellen ${ }^{38}$ ) als Ausgangsmaterial zur Herstellung von Organoiden, die für den klinischen Einsatz (einschließlich klinischer Versuche ${ }^{39}$ ) im oder am menschlichen Körper bestimmt sind, den Regelungen des TPG unterliegen. Die Entnahme beim lebenden Spender muss daher den Anforderungen der §§ 8, 8b TPG genügen, während für die Entnahme bei toten Spendern den §§ 3 ff. TPG Rechnung zu tragen ist. § 8d TPG enthält zudem in Verbindung mit der TPG-Gewebeverordnung nähere Anforderungen an die Einrichtung, die das Gewebe zum Zwecke der Übertragung entnimmt, untersucht, aufbereitet, be- oder verarbeitet, konserviert, kennzeichnet, verpackt, aufbewahrt oder an andere abgibt ( $\$ 1 \mathrm{a}$ Nr. 8 TPG). Von besonderer Bedeutung ist schließlich, dass mit den Geweben kein Handel getrieben werden darf ( $\$ 17$ TPG). ${ }^{40}$

Das TPG findet dagegen keine Anwendung, wenn die somatischen Zellen oder Organoide zu Forschungszwecken nur im Labor verwendet werden sollen, da es hier zum Zeitpunkt der Entnahme am Übertragungszweck fehlt. ${ }^{41}$ Auch ist die Xenotransplantation vom Anwendungsbereich des TPG nicht erfasst. ${ }^{42}$

\subsubsection{Arzneimittelgesetz}

§ 20 b Abs. 1 S. 1 Arzneimittelgesetz (AMG) ${ }^{43}$ verlangt grundsätzlich ${ }^{44}$ das Einholen einer Erlaubnis der zuständigen Landesbehörde für die Gewinnung von zur Verwendung bei Menschen bestimmtem Gewebe i. S. d. § 1a Nr. 4 TPG. ${ }^{45}$ Gewinnung in diesem Sinne ist die direkte oder extrakorporale Entnahme von Gewebe einschließlich aller

36 Gewebegesetz vom 20. Juli 2007 (BGBl. I: 1574), geändert durch Artikel 17 des Gesetzes vom 9. August 2019 (BGBl. I: 1202).

37 BT-Drucks. 16/3146: 21.

38 Aber ausgenommen Blut und Blutbestandteile, siehe Gerke, 2020a: $296 \mathrm{ff}$.

39 BT-Drucks. 16/3146: 23.

40 Vgl. Wernscheid, 2012: 229.

41 BT-Drucks. 16/3146: 23.

42 Krüger et al., 2009: $62 \mathrm{f}$.

43 Arzneimittelgesetz in der Fassung der Bekanntmachung vom 12. Dezember 2005 (BGBl. I: 3394), zuletzt geändert durch Artikel 3c des Gesetzes vom 10. Februar 2020 (BGBl. I: 148).

44 Ausnahmen in $\S 20$ b Abs. 2, 20 d S. 1 AMG.

45 Wernscheid, 2012: 116; näher zur Erlaubnis nach § 20b AMG siehe Gerke, 2020a: 284 ff.; Gerke et al., 2020b: 440. 
Maßnahmen, die dazu bestimmt sind, das Gewebe in einem be- oder verarbeitungsfähigen Zustand zu erhalten, eindeutig zu identifizieren und zu transportieren (Satz 2). Die Erlaubnis darf nach Satz 3 nur versagt werden, „wenn

1. eine angemessen ausgebildete Person mit der erforderlichen Berufserfahrung (verantwortliche Person nach § 20b) nicht vorhanden ist, die, soweit es sich um eine Entnahmeeinrichtung handelt, zugleich die ärztliche Person im Sinne von §8d Abs. 1 Satz 1 des Transplantationsgesetzes sein kann,

2. weiteres mitwirkendes Personal nicht ausreichend qualifiziert ist,

3. angemessene Räume für die jeweilige Gewebegewinnung oder für die Laboruntersuchungen nicht vorhanden sind,

4. nicht gewährleistet wird, dass die Gewebegewinnung oder die Laboruntersuchungen nach dem Stand der medizinischen Wissenschaft und Technik und nach den Vorschriften der Abschnitte 2, 3 und 3a des Transplantationsgesetzes vorgenommen werden, oder

5. die verantwortliche Person nach § 20 b oder der Antragsteller die zur Ausübung ihrer oder seiner Tätigkeit erforderliche Zuverlässigkeit nicht besitzt.“

\subsubsection{Tierschutzgesetz}

Sollen die zur Herstellung von Organoiden benötigten Zellen aus Tieren gewonnen werden, ist das Tierschutzgesetz (TierSchG) zu beachten. ${ }^{46}$ Es schützt grundsätzlich alle Tiere, enthält aber Differenzierungen seines Schutzumfangs: Die meisten Einzelbestimmungen beziehen sich nur auf Wirbeltiere.

$\S 6$ Abs. 1 S. 1 TierSchG verbietet grundsätzlich (vorbehaltlich der in $\S 6$ enthaltenen Ausnahmen) das vollständige oder teilweise Amputieren von Körperteilen oder das vollständige oder teilweise Entnehmen oder Zerstören von Organen oder Geweben eines Wirbeltieres. Das Entnahmeverbot verbietet (sub „Gewebe“) auch das Entnehmen oder Zerstören einzelner Zellen, sofern dadurch die Funktion (= spezifische Leistung) eines ganzen Zellverbandes (= Gewebes) beeinträchtigt wird. ${ }^{47}$ Das wird bei der Herstellung von Organoiden selten der Fall sein. Das Entnahmeverbot ist daher in der

46 Tierschutzgesetz in der Fassung der Bekanntmachung vom 18. Mai 2006 (BGBl. I: 1206, 1313), zuletzt geändert durch Artikel 101 des Gesetzes vom 20. November 2019 (BGBl. I: 1626).

47 Hirt et al., 2016: § 6 TierSchG Rdnr. 1. 
Regel nicht einschlägig. ${ }^{48}$ Gemäß § 6a TierSchG sind zudem die Vorschriften zu Tierversuchen spezieller, sodass das Verbot des § 6 TierSchG ohnehin nur für standardisierte Verfahren gilt, die nicht mehr als Tierversuch eingestuft werden können.

Eingriffe oder Behandlungen, durch die Organe oder Gewebe ganz oder teilweise entnommen werden, um zu wissenschaftlichen Zwecken Kulturen anzulegen oder isolierte Organe, Gewebe oder Zellen zu untersuchen, gelten nach $\S 7$ Abs. 2 TierSchG ebenfalls als Tierversuche.

\subsection{Einordnung der hergestellten Organoide}

\subsubsection{Organoide als menschliche Lebewesen}

Zahlreiche Rechtsfragen würden heraufbeschworen, wenn die erzeugten Entitäten eine Entwicklungsfähigkeit wie menschliche Embryonen aufweisen würden, etwa als sogenannte Embryoide (siehe zu Embryoiden auch Nicolas/Etoc/Brivanlou, Kap. 5). ${ }^{49}$ Denn dann könnte ihnen unter Umständen Menschenwürde (Art. 1 GG) und Lebensschutz (Art. 2 Abs. 1 GG) zuzuweisen sein. Allerdings wäre ihre Herstellung allenfalls dann vom ESchG erfasst und damit verboten, wenn sie die gleiche Erbsubstanz wie ein anderer Embryo, ein Foetus, ein Mensch oder ein Verstorbener aufweisen würden. Denn dann könnte unter Umständen ein Verstoß gegen § 6 ESchG (Verbot des Klonens) bejaht werden. ${ }^{50}$ Auch wäre ihre Verwendung für Forschungszwecke möglicherweise nach § 2 Abs. 1 ESchG verboten. Jedenfalls rechtspolitisch wäre allerdings zu überlegen, ob ein so starker rechtlicher Schutz wegen der im Vergleich zu natürlichen Embryonen andersartigen Art der Herstellung unter Vermeidung einer Befruchtung, wegen der Entstehung in einem völlig anderen Kontext als der Erzeugung von Nachkommen und ggf. auch wegen der Absicht, die Entwicklung der entsprechenden Entitäten in einem sehr frühen Stadium $\mathrm{zu}$ beenden, $\mathrm{zu}$ verneinen wäre. ${ }^{51}$ Andere Vor-

48 In Betracht kommen kann ferner die Ausnahme gemäß $\S 6$ Abs. 1 S. 2 Nr. 4 TierSchG, sofern die Gewebeentnahme anderen als wissenschaftlichen Zwecken, vielmehr insbesondere der Heilbehandlung von Menschen oder Tieren dient. Sofern es sich um ein noch im Versuch befindliches Verfahren handelt, sind die §§ 7-10 über Tierversuche einschlägig, siehe § 7 Abs. 2 TierSchG.

49 Siehe dazu Stein, 2019; Denker, 2017: 15 ff.; Munsie et al., 2017: 943.

50 Allerdings ist sehr umstritten, inwiefern es für die Anwendbarkeit des Klonierungsverbots auf die Art der Herstellung der entsprechenden Entität ankommt, siehe Günther, 2014: C. II. § 6 Rdnr. 3 ff.; Taupitz, 2014: C. II. § 8 Rdnrn. $48 \mathrm{ff}$.

51 Vgl. dazu Taupitz, 2001: 3440; ähnlich später Deutscher Ethikrat, 2011: 100; weitere Nachweise zu entsprechenden Überlegungen in der angelsächsischen Literatur im Hinblick auf den moralischen Status früher Embryonen bei Hostiuc et al., 2019: 119. 
schriften, die die Herstellung von Embryonen reglementieren (z. B. § 1 Abs. 1 Nr. 2 ESchG), wären jedenfalls mangels einer von ihnen ausdrücklich vorausgesetzten Befruchtung sicher nicht einschlägig. Auch § 7 ESchG, der die Herstellung von Hybriden und Chimären regelt, würde nicht zu einem Verbot führen, sofern nicht Embryonen mit unterschiedlicher Erbinformation unter Verwendung mindestens eines menschlichen Embryos zu einem Zellverband vereinigt werden (§ 7 Abs. 1 Nr. 1 ESchG), nicht mit einem menschlichen Embryo eine Zelle verbunden wird, die eine andere Erbinformation als die Zellen des Embryos enthält und sich mit diesem weiter zu differenzieren vermag (§ 7 Abs. 1 Nr. 2 ESchG), und auch keine Befruchtung stattfindet (§ 7 Abs. 1 Nr. 3 ESchG). ${ }^{52}$

Vom ESchG (oder vom sonstigen deutschen Recht) nicht ansatzweise geklärt und auch rechtspolitisch offen ist die Frage, ob weit entwickelte Hirnorganoide, wie sie in der Zukunft einmal möglich sein könnten, denselben Regeln wie Embryonen unterliegen sollten (siehe zu Hirnorganoiden näher Tanaka/Park, Kap. 3.5, und Schicktanz, Kap. 6). Dies könnte gemäß einer in der Literatur vertretenen Auffassung dann bejaht werden, wenn man den Beginn des „Hirnlebens“ (etwa am 57. Tag post conceptionem) als entscheidende Zäsur für den vollen rechtlichen Schutz des werdenden menschlichen Lebens annimmt. ${ }^{53}$ Weiter vorverlagert, aber letztlich in dieselbe Richtung gehend, wird die in anderen Rechtsordnungen geltende sogenannte 14-Tage-Regel (keine In-vitro-Entwicklung von Embryonen über den 14. Tag hinaus) gelegentlich daran geknüpft, dass etwa am 14. Tag der embryonalen Entwicklung mit dem Auftreten des Primitivstreifens die ersten Anzeichen eines sich ausbildenden Nervensystems und damit des Gehirns entstehen. ${ }^{54}$ Wenn der moralische und auch der rechtliche Status von Embryonen in dieser Form mit der beginnenden Gehirnentwicklung verknüpft wird, könnte es naheliegen, die Herstellung weit entwickelter zerebraler Organoide bzw. die Forschung mit ihnen zu verbieten. ${ }^{55}$

Die Herstellung von embryoähnlichen Organoiden und auch von zerebralen Organoiden, die einem funktionsfähigen menschlichen Gehirn auch nur ansatzweise gleichen, ist freilich allenfalls Zukunftsmusik. Ein wirklicher Handlungsbedarf besteht für den Gesetzgeber zurzeit nicht.

52 Siehe zu § 7 ESchG näher Günther, 2014: C. II. § 7; Deutscher Ethikrat, 2011: 38 ff.

53 So insbesondere Sass, 1989: $160 \mathrm{ff}$.; weitere Nachweise bei Müller-Terpitz, 2008: $182 \mathrm{ff}$.

54 Hostiuc et al., 2019: 119.

55 Siehe etwa Hostiuc et al., 2019: $119 \mathrm{ff}$. 


\subsubsection{Organoide als Sachen}

Sofern Organoide nicht die Entwicklungsfähigkeit von Embryonen, also von gesamten Individuen aufweisen, sondern lediglich einzelnen Organen ähneln, sind sie nicht anders zu behandeln als (andere) menschliche Organe oder Zellstrukturen. Sie haben keinen besonderen rechtlichen „Status“. Es handelt sich nach deutschem Recht um Sachen, an denen Eigentum nach $\S \S 903$ ff. BGB (Bundesgesetzbuch) bestehen kann, ${ }^{56}$ wobei in der Regel der Forscher durch „Verarbeitung oder Umbildung eines oder mehrerer Stoffe [der Ausgangszellen] eine neue bewegliche Sache“ (das Organoid) hergestellt haben wird und er deshalb in der Regel gemäß § 950 BGB originär das Eigentum daran erwirbt. Sofern sich genetisches Material des Spenders der Ausgangszellen in dem Organoid fortsetzt, erstreckt sich allerdings auch das Allgemeine Persönlichkeitsrecht des Spenders auf das Organoid. ${ }^{57}$ Damit hat der Spender - wie oben dargestellt - das Recht, über die Verwendung des Organoids zu bestimmen. Da die Übertragung des Organoids - wie sogleich darzustellen sein wird - nicht den Regeln des Transplantationsgesetzes unterliegt, ist das Bestimmungsrecht des Spenders der Ausgangszellen auch nicht etwa durch das öffentlich-rechtliche Organverteilungsverfahren gemäß § 12 TPG beschränkt. Zudem sei betont, dass für die hergestellten Organoide wegen $§ 17 \mathrm{Abs.} 1$ S. 2 Nr. 2 TPG kein Verbot des Organhandels gilt. ${ }^{58}$

\subsection{Umgang mit Organoiden / Verwendung der Organoide}

\subsubsection{Transplantationsgesetz}

Sofern die hergestellten Organoide zur Übertragung auf andere Menschen bestimmt sind, könnte auf diesen Vorgang wiederum das Transplantationsgesetz anwendbar sein. Denn neben der „Spende und [...] Entnahme von menschlichen Organen oder Geweben zum Zwecke der Übertragung“" (siehe dazu oben 7.2.4) gilt das TPG nach seinem $\S 1$ Abs. 2 S. 1 ebenfalls „für die Übertragung der Organe oder der Gewebe einschließlich der Vorbereitung dieser Maßnahmen“.

Organoide sind Organe im Sinne des § 1a Nr. 1 TPG, sofern sie „mit Ausnahme der Haut, [...] aus verschiedenen Geweben bestehende [...], differenzierte [...] Teile des

56 Siehe oben 2.3. Zur internationalen Diskussion siehe etwa die Nachweise bei Boers et al., 2016.

57 Siehe oben 2.3.

58 Vgl. zum Tissue Engineering Wernscheid, 2012: 129f., 221. 
menschlichen Körpers [sind], die in Bezug auf Struktur, Blutgefäßversorgung und Fähigkeit zum Vollzug physiologischer Funktionen eine funktionale Einheit bilden“ ". 59

Legt man das Tatbestandsmerkmal „Teil des menschlichen Körpers“ eng aus, so würden nur solche Bestandteile erfasst, die auf natürliche Weise im Körper eines Menschen vorhanden sind. Danach wären in vitro („künstlich“) hergestellte Organoide keine Organe im Sinne des TPG. Für diese enge Auslegung enthält der Wortlaut des TPG allerdings keine Anhaltspunkte. Er unterscheidet nicht zwischen natürlich vorhandenen und künstlich hergestellten Bestandteilen. Das Tatbestandsmerkmal „Teile des menschlichen Körpers" ist damit dahingehend auszulegen, dass auch künstlich hergestellte Teile menschlichen Ursprungs erfasst sind.$^{60}$ Dafür spricht auch, dass das TPG in seiner Ausgestaltung durch das Gewebegesetz den Gefahren entgegenwirken soll, die durch eine ungeregelte Gewinnung, Verarbeitung, Lagerung und Verteilung von Zellen und Geweben entstehen, wie beispielsweise einer Kontamination und der Übertragung von Krankheiten. ${ }^{61}$ Auch bei artifiziell erzeugten Geweben, die sich aus einzelnen menschlichen Zellen zusammensetzen, bestehen diese Risiken. ${ }^{62}$

Gleichwohl finden die Übertragungsvorschriften des TPG keine Anwendung auf Organoide. Das gilt ungeachtet der Tatsache, dass Organoide noch weit davon entfernt sind, ,in Bezug auf Struktur, Blutgefäßversorgung und Fähigkeit zum Vollzug physiologischer Funktionen eine funktionale Einheit zu bilden“. Denn zum einen nimmt § $1 \mathrm{a}$ Nr. 1 TPG seit der 15. AMG-Novelle (2009) Gewebe (und damit auch einzelne Zellen), die im Rahmen einer Bearbeitung zur Herstellung von Arzneimitteln für neuartige Therapien im Sinne des $\S 4$ Abs. 9 des AMG bestimmt sind, aus dem Anwendungsbereich des TPG aus; sie sind vielmehr dem Erfordernis einer Herstellungserlaubnis und einer Zulassung nach Arzneimittelrecht unterstellt. ${ }^{63}$ Das ist, wie zu zeigen sein wird (unten 4.2), bezüglich der Herstellung von Organoiden der Fall.

Darüber hinaus heißt es in § 1 Abs. 2 S. 1 TPG: „Dieses Gesetz gilt für die Spende und die Entnahme von menschlichen Organen oder Geweben zum Zwecke der Übertragung

59 Dazu gehören nach § 1a Nr. 1 TPG auch Organteile und einzelne Gewebe eines Organs, die unter Aufrechterhaltung der Anforderungen an Struktur und Blutgefäßversorgung zum gleichen Zweck wie das ganze Organ im menschlichen Körper verwendet werden können, mit Ausnahme solcher Gewebe, die zur Herstellung von Arzneimitteln für neuartige Therapien im Sinne des § 4 Abs. 9 des Arzneimittelgesetzes bestimmt sind.

60 Gerke, 2020a: 291; zur österreichischen Rechtslage ebenso Kopetzki et al., 2020: $362 \mathrm{f}$.

61 BT-Drucks. 16/3146, 2006: 21.

62 Gerke, 2020a: 291; zur österreichischen Rechtslage ebenso Kopetzki et al., 2020: 363.

63 Näher Wernscheid, 2012: 139 f.; Gerke, 2020a: 263, 284 f. 
sowie für die Übertragung der Organe oder der Gewebe“, 64 nicht aber „von“ Organen oder Geweben. Die Organoide ihrerseits sind aber nicht das Resultat einer Spende oder Entnahme von menschlichen Organen i. S. d. § 1a Nr. 1 TPG. ${ }^{65}$ Es mangelt an der Entnahme eines funktionstüchtigen und übertragungsfähigen Organs oder Organteils aus dem Körper eines Spenders. Vielmehr findet in solchen Fällen lediglich eine Implantation eines durch einen Herstellungsprozess im Labor gezüchteten Organkomplexes statt. Auch die Voraussetzung, dass vor und nach der Entnahme dieselbe Funktion im menschlichen Körper erfüllt wird, kann bei Organoiden mangels ursprünglich bestehender Organfunktion nicht gegeben sein. ${ }^{66}$

Herz-, Nieren-, Leber-, Lungen-, Darm- und Bauchspeicheldrüsen-Organoide wären erst recht keine vermittlungspflichtigen Organe i. S. d. § 1a Nr. 2 TPG, da sie nicht nach $\S 3$ TPG oder § 4 TPG einem toten Spender entnommen wurden.

Allerdings müsste die Allokation der derzeit vermittlungspflichtigen Organe (vgl. $\S 12$ TPG) wohl neu geregelt werden, wenn tatsächlich eines Tages entsprechende Organoide verfügbar wären. ${ }^{67}$ Hierbei müsste geklärt werden, wer ein künstlich hergestelltes Organ oder stattdessen ein von einem toten Spender entnommenes Organ erhalten soll. Vielleicht können in Zukunft auch derart viele Organoide hergestellt werden, dass das Problem des Mangels an Organen dann nicht mehr besteht.

\subsubsection{Arzneimittelgesetz}

Soweit Organoide zur „Anwendung im oder am menschlichen oder tierischen Körper bestimmt" sind, ist möglicherweise das Arzneimittelrecht anwendbar. Denn, wie zu zeigen sein wird, gilt das Arzneimittelrecht auch für zellbasierte Stoffe.

Die zentrale Rechtsgrundlage im Arzneimittelrecht ist das AMG, ${ }^{68}$ mit dem unter anderem die Richtlinie (RL) 2001/83/EG ${ }^{69}$ umgesetzt wurde. Daneben sind die unmittelbar anwendbaren Verordnungen der EU einschlägig. Hierzu gehören aus dem Blickwinkel des vorliegenden Beitrags insbesondere die Verordnung (VO) (EG) 1394/2007

64 Hervorhebung vom Verfasser.

65 Vgl. Gerke, 2020a: 295; ferner (für das österreichische Recht) Kopetzki et al., 2020: 374.

66 Vgl. ebd.

67 Siehe Gerke, 2020a: 292.

68 Siehe Fn. 43.

69 Richtlinie 2001/83/EG des Europäischen Parlaments und des Rates vom 6. November 2001 zur Schaffung eines Gemeinschaftskodexes für Humanarzneimittel. In: Amtsblatt der Europäischen Union L 2001/311: 67 . 
$\left(\right.$ ATMP-VO) ${ }^{70}$ und die VO (EG) 726/2004..$^{71}$ Denn Organoide können Arzneimittel für neuartige Therapien (Advanced Therapy Medicinal Products, ATMP) sein. ATMP sind im Ausgangspunkt Arzneimittel, sodass diese Produkte auch die grundsätzlichen Arzneimitteleigenschaften aufweisen müssen. Der Unterschied zu anderen Arzneimitteln besteht in der Grundsubstanz, aus denen ATMP hergestellt werden, sowie in der Art und Weise ihrer Herstellung.

Zur Übertragung auf einen Menschen bestimmte Organoide sind „Stoffe“ i. S. d. \& 3 AMG. Sie haben eine therapeutische Zweckbestimmung („,zur Heilung oder Linderung oder zur Verhütung [...] menschlicher Krankheiten oder krankhafter Beschwerden bestimmt“, 2 Abs. 1 Nr. 1 AMG). Da Organoide lebensfähige Zellen enthalten oder aus ihnen bestehen, kann es auch sein, dass sie bei der Wiederherstellung, Beeinflussung oder Korrektur der menschlichen physiologischen Funktionen gemäß $§ 2$ Abs. 1 Nr. 2 AMG hauptsächlich pharmakologisch, immunologisch und/oder metabolisch wirken. ${ }^{72}$ Sie sind damit Arzneimittel im Sinne des AMG.

ATMP sind sie, wenn sie einer der vier Gruppen von ATMP zuzuordnen sind ( $\$ 4$ Abs. 9 AMG). Dazu gehören Gentherapeutika, somatische Zelltherapeutika, biotechnologisch bearbeitete Gewebeprodukte oder kombinierte ATMP. ${ }^{73}$ In der Regel sind Organoide biotechnologisch bearbeitete Gewebeprodukte i. S. d. Art. 2 Abs. 1 lit. b ATMPVo, da sie zum einen „biotechnologisch bearbeitete Zellen oder Gewebe“ enthalten oder aus ihnen bestehen (Spiegelstrich 1) und zum anderen ihnen gerade „Eigenschaften zur Regeneration, Wiederherstellung oder zum Ersatz menschlichen Gewebes zugeschrieben werden“ oder sie „zu diesem Zweck [...] Menschen verabreicht“ werden (Spiegelstrich 2).

Organoide dürften zudem immer substanziell bearbeitete Zellen oder Gewebe i. S. d. Art. 2 Abs. 1 lit. c Spiegelstrich 1 ATMP-VO enthalten, das heißt die Zellen oder Gewebe werden dergestalt „substanziell bearbeitet“ worden sein, „dass biologische Merkmale,

70 Verordnung (EG) 1394/2007 des Europäischen Parlaments und des Rates vom 13. November 2007 über Arzneimittel für neuartige Therapien und zur Änderung der Richtlinie 2001/83/EG und der Verordnung (EG) 726/2004. In: Amtsblatt der Europäischen Union L 2007/324: 121.

71 Verordnung (EG) 726/2004 des Europäischen Parlaments und des Rates vom 31. März 2004 zur Festlegung von Unionsverfahren für die Genehmigung und Überwachung von Human- und Tierarzneimitteln und zur Errichtung einer Europäischen Arzneimittel-Agentur. In: Amtsblatt der Europäischen Union L 2004/136: 1.

72 Vgl. auch Art. 2 Abs. 2 ATMP-VO. Vgl. entsprechend zu Produkten basierend auf humanen induzierten Stammzellen Gerke, 2020a: $253 \mathrm{ff}$.

73 Vgl. zum Folgenden Faltus, 2016: 693 ff.; Gerke, 2020a: $247 \mathrm{ff}$. 
physiologische Funktionen oder strukturelle Eigenschaften, die für die beabsichtigte Regeneration, Wiederherstellung oder den Ersatz relevant sind, erzielt werden“.

Als ATMP unterliegen Organoide grundsätzlich der zentralen Zulassung auf europäischer Ebene. ATMP dürfen gemäß Art. 3 Abs. 1 und Anhang Nr.1a VO (EG) 726/2004 innerhalb der EU nur in Verkehr gebracht werden, wenn von der Europäischen Kommission eine Genehmigung für das Inverkehrbringen erteilt worden ist. Der Antrag für die Genehmigung für das Inverkehrbringen eines ATMP ist bei der EMA einzureichen. ${ }^{74}$ Voraussetzung für die Erteilung der Genehmigung ist grundsätzlich eine klinische Prüfung nach $\S \S 40 \mathrm{ff}$. AMG. ${ }^{75}$

ATMP können aber auch unter die sogenannte Krankenhausausnahme nach $\S 4 \mathrm{~b}$ AMG fallen. § 4b AMG stellt die nationale Umsetzung des Art. 3 Nr. 7 RL 2001/83/EG dar (vgl. auch Art. 28 Nr. 2 ATMP-VO). Nach $₫ 4$ b Abs. 1 S. 1 AMG sind solche ATMP von der Krankenhausausnahme erfasst, die in Deutschland

„1. als individuelle Zubereitung für einen einzelnen Patienten ärztlich verschrieben, 2. nach spezifischen Qualitätsnormen nicht routinemäßig hergestellt[ $\left.{ }^{76}\right]$ und

3. in einer spezialisierten Einrichtung der Krankenversorgung unter der fachlichen Verantwortung eines Arztes angewendet werden“.

Für Organoide, die unter die Krankenhausausnahme nach $\S 4 \mathrm{~b}$ AMG fallen, finden weder der Vierte Abschnitt (Zulassung der Arzneimittel) ${ }^{77}$ noch der Sechste (Schutz des Menschen bei der klinischen Prüfung) ${ }^{78}$ oder der Siebte Abschnitt (Abgabe von Arzneimitteln) des AMG Anwendung. ${ }^{79}$ Insbesondere bedürfen sie keiner zentralen Zulassung

74 Vgl. Art. 4 Abs. 1 Vo (EG) 726/2004.

75 Vgl. Paul-Ehrlich-Institut, 2012: 20; Gerke, 2020a: 280 ff. Zu den Herausforderungen, die klinische Prüfungen mit Organoiden stellen, siehe Boers et al., 2016: 941 . Zu beachten ist die Besonderheit des $\S 40$ Abs. 1 S. 3 Nr. 2a AMG bezüglich der klinischen Prüfung eines Arzneimittels (einschließlich eines ATMP), das aus einem GVO oder einer Kombination von GVO besteht oder solche enthält (GVO-haltiges Arzneimittel bzw. GVO-haltiges ATMP).

76 Der Begriff „nicht routinemäßig hergestellt“ in § 4b Abs. 1 S. 1 Nr. 2 AMG wird in $\S 4$ b Abs. 2 AMG definiert und erfasst ,insbesondere Arzneimittel,

1. die in so geringem Umfang hergestellt und angewendet werden, dass nicht zu erwarten ist, dass hinreichend klinische Erfahrung gesammelt werden kann, um das Arzneimittel umfassend bewerten zu können, oder

2. die noch nicht in ausreichender Anzahl hergestellt und angewendet worden sind, sodass die notwendigen Erkenntnisse für ihre umfassende Bewertung noch nicht erlangt werden konnten“.

77 Mit Ausnahme des § 33 AMG (Gebühren und Auslagen).

78 Gerke, 2020a: 277.

79 Vgl. § 4b Abs. 1 S. 1 AMG. 
auf europäischer Ebene. Die übrigen Vorschriften des AMG wie insbesondere zur Herstellungserlaubnis ( $\$ 13 \mathrm{AMG}^{80}$ ), zur Abgabe von ATMP an andere ( $\S 4 \mathrm{~b}$ Abs. 1 S. 1 , Abs. 3 S. 1 AMG), zur Pharmakovigilanz gemäß Art. 14 Abs. 1 ATMP-VO und zur Rückverfolgbarkeit gemäß Art. 15 Abs. 1-6 ATMP-VO gelten aber entsprechend. ${ }^{81}$

\subsubsection{Gentechnikgesetz}

Je nach Art ihrer Herstellung oder Behandlung können Organoide gentechnisch veränderte Organismen sein mit der Folge, dass das Gentechnikgesetz (GenTG) einschlägig ist. $^{82}$ Nach § 2 Abs. 1 GenTG gilt das Gesetz für „gentechnische Anlagen“ (Nr. 1), „gentechnische Arbeiten“ (Nr.2), „Freisetzungen von gentechnisch veränderten Organismen“ (GVO) (Nr. 3) und „das Inverkehrbringen von Produkten, die [...] [GVO] enthalten oder aus solchen bestehen“ (Nr. 4). Allerdings gilt das GenTG nach seinem § 2 Abs. 3 nicht für die unmittelbare Anwendung von GVO am Menschen. Die Humangenetik ist vom Anwendungsbereich des GenTG ausgenommen. Daher fällt beispielsweise die somatische Gentherapie unter unmittelbarer Anwendung von GVO am Menschen nicht in den Anwendungsbereich des GenTG. ${ }^{83}$ Das Gesetz erfasst allerdings die In-vitro-Teilschritte der Verfahren, die der unmittelbaren Anwendung von GVO am Menschen voroder nachgelagert sind. ${ }^{84}$

\footnotetext{
80 Vgl. Gerke et al., 2020b: 277. Die Erlaubnispflicht nach § 13 Abs. 1 S. 1 Nr. 1 AMG erstreckt sich auf die berufs- oder gewerbsmäßige Herstellung von Arzneimitteln i. S. v. § 2 Abs. 1 und Abs. 2 Nr. 1 AMG und somit ebenfalls von ATMPs i. S. d. § 4 Abs. 9 AMG (i. V. m. Art. 2 Abs. 1 lit. a ATMP-VO). Die Erlaubnispflicht erstreckt sich auch auf die gewerbs- oder berufsmäßige Herstellung von ATMPs, die unter die Krankenhausausnahme nach § 4b AMG fallen (vgl. § 4b Abs. 1 S. 2 AMG [,die übrigen Vorschriften des Gesetzes (...) gelten entsprechend“] i. V. m. § 4b Abs. 1 S. 1 AMG). Auch Art. 3 Nr. 7 RL 2001/83/EG, der durch Art. 28 Nr. 2 ATMP-VO eingefügt und in § $4 \mathrm{~b}$ AMG national umgesetzt wurde, sieht explizit das Erfordernis einer Herstellungserlaubnis vor.

81 Vgl. § 4b Abs. 1 S. 2 AMG; näher Wernscheid, 2020: 131 ff.; Gerke, 2020a: 2.7.2.

82 Vgl. Gerke, 2020a: $304 \mathrm{ff}$.

83 Fenger, 2018: § 2 GenTG Rn. 4.; Gerke, 2020a: 304.

84 Fenger, 2018: § 2 GenTG Rn. 5; Gerke, 2020a: 304.
} 


\subsection{4 Ärztliches Berufsrecht}

Das ärztliche Berufsrecht, konkret: das Satzungsrecht der Ärztekammern, enthält folgende Regelung: 85

„Ärztinnen und Ärzte, die sich an einem Forschungsvorhaben beteiligen, bei dem in die psychische oder körperliche Integrität eines Menschen eingegriffen oder Körpermaterialien oder Daten verwendet werden, die sich einem bestimmten Menschen zuordnen lassen, müssen sicherstellen, dass vor der Durchführung des Forschungsvorhabens eine Beratung erfolgt, die auf die mit ihm verbundenen berufsethischen und berufsrechtlichen Fragen zielt und die von einer bei der zuständigen Ärztekammer gebildeten Ethik-Kommission oder von einer anderen, nach Landesrecht gebildeten unabhängigen und interdisziplinär besetzten Ethik-Kommission durchgeführt wird. Dasselbe gilt vor der Durchführung gesetzlich zugelassener Forschung mit vitalen menschlichen Gameten und lebendem embryonalen Gewebe."

Im Ergebnis unterliegt die Herstellung von Organoiden (abgesehen von den Vorschriften des AMG zu klinischen Prüfungen ${ }^{86}$ ) nur dann einer Bewertung durch eine Ethikkommission, wenn ein Arzt daran beteiligt ist und entweder lebendes embryonales Gewebe oder Körpermaterialien oder Daten verwendet werden, die sich einem bestimmten Menschen zuordnen lassen.

\subsubsection{Tierschutzgesetz}

Im Interesse der betroffenen lebenden Tiere enthält das Tierschutzgesetz (TierSchG) Regeln für die Durchführung eines Tierversuchs. Ein Tierversuch liegt in Abgrenzung $\mathrm{zu}$ anderen Maßnahmen dann vor, wenn das Verfahren noch nicht zur Praxisreife entwickelt ist und sein Versuchscharakter im Vordergrund steht. ${ }^{87}$

Tierversuche sind grundsätzlich genehmigungspflichtig ( 8 TierSchG). Soweit sie rechtlich vorgeschrieben sind, etwa im Rahmen der Arzneimittelzulassung, sind sie lediglich anzeigepflichtig (§ 8a TierSchG). Die Genehmigung wird durch eine Behörde erteilt; zuvor muss eine interdisziplinär zusammengesetzte Kommission (in Anlehnung

85 Siehe stellvertretend für die Berufsordnungen der Landesärztekammern § 15 der Musterberufsordnung für die in Deutschland tätigen Ärztinnen und Ärzte von 2018 unter: https://www.bundesaerz tekammer.de/recht/berufsrecht/muster-berufsordnung-aerzte/ [31.3.2020].

86 Siehe Fn. 75.

87 Hirt et al., 2016: § 7 TierSchG Rdnr. 6; Deutscher Ethikrat, 2011: 47. 
an entsprechende medizinische Kommissionen häufig als „Ethikkommission“ bezeichnet) Stellung zu dem Tierversuch genommen haben (§ 15 TierSchG). ${ }^{88}$

§ 7 Abs. 2 S. 1 TierSchG definiert den Tierversuch als „Eingriffe oder Behandlungen zu Versuchszwecken

1. an Tieren, wenn sie mit Schmerzen, Leiden oder Schäden für diese Tiere verbunden sein können,

2. an Tieren, die dazu führen können, dass Tiere geboren werden oder schlüpfen, die Schmerzen, Leiden oder Schäden erleiden, oder

3. am Erbgut von Tieren, wenn sie mit Schmerzen, Leiden oder Schäden für die erbgutveränderten Tiere oder deren Trägertiere verbunden sein können.“

Geschützter Versuchsgegenstand ist bei § 7 Abs. 2 S. 1 Nr. 1 TierSchG nur das lebende geborene Tier. ${ }^{89} \S 7$ Abs. 2 S. 1 Nr. 3 TierSchG schützt dagegen das Erbgut des Tieres, und zwar im Interesse des davon betroffenen (später) geborenen Tieres. ${ }^{90}$ Erfasst sind daher anders als bei Nr. 1 auch Eingriffe an Eizellen und Embryonen. Die möglichen Folgen des Versuchs müssen aber für das erbgutveränderte oder das zur Austragung verwendete Tier drohen. ${ }^{91}$

Als Tierversuche gelten nach § 7 Abs. 2 S. 2 Nr. 2 TierSchG auch Eingriffe oder Behandlungen,

„2. durch die Organe oder Gewebe ganz oder teilweise entnommen werden, um zu wissenschaftlichen Zwecken[ $\left.{ }^{92}\right]$

a) die Organe oder Gewebe zu transplantieren,

b) Kulturen anzulegen oder

c) isolierte Organe, Gewebe oder Zellen zu untersuchen [...]“.

Gerade diese Bestimmungen können im Fall von Organoiden einschlägig sein.

88 Hirt et al., 2016: § 15 TierSchG Rdnr. 4.

89 Hirt et al., 2016: § 7 TierSchG Rdnr. 6.

90 Ebd.

91 Deutscher Ethikrat, 2011: 48.

92 Diese liegen dann vor, wenn es um einen Erkenntnisgewinn zu einem noch nicht hinreichend gelösten wissenschaftlichen Problem geht, siehe Hirt et al., 2016: § 7 TierSchG Rdnr. 11. 
Tierversuche sind aus dem Blickwinkel des 3-R-Prinzips („Replace, Reduce, Refine“, also „Vermeiden, Vermindern, Verbessern“) ${ }^{93}$ nach § 7 Abs. 1 S. 2 TierSchG „im Hinblick auf

a) die den Tieren zuzufügenden Schmerzen, Leiden und Schäden,

b) die Zahl der verwendeten Tiere,

c) die artspezifische Fähigkeit der verwendeten Tiere, unter den Versuchseinwirkungen zu leiden, auf das unerlässliche Maß zu beschränken“ und

„die Tiere, die zur Verwendung in Tierversuchen bestimmt sind oder deren Gewebe oder Organe dazu bestimmt sind, zu wissenschaftlichen Zwecken verwendet zu werden, [sind] so zu halten, zu züchten und zu pflegen, dass sie nur in dem Umfang belastet werden, der für die Verwendung zu wissenschaftlichen Zwecken unerlässlich ist."

Tierversuche dürfen gemäß $\S 7 \mathrm{a}$ Abs. 1 TierSchG ferner nur durchgeführt werden, wenn sie zu einem gesetzlich näher bestimmten Zweck unerlässlich sind. Als zulässige Versuchszwecke gelten insbesondere

„1. Grundlagenforschung,

2. sonstige Forschung mit einem der folgenden Ziele:

a) Vorbeugung, Erkennung oder Behandlung von Krankheiten, Leiden, Körperschäden oder körperlichen Beschwerden bei Menschen oder Tieren,

b) Erkennung oder Beeinflussung physiologischer Zustände oder Funktionen bei Menschen oder Tieren“.

§ 7a Abs. 2 TierSchG konkretisiert die Unerlässlichkeit wie folgt: „Bei der Entscheidung, ob ein Tierversuch unerlässlich ist, sowie bei der Durchführung von Tierversuchen sind folgende Grundsätze zu beachten:

1. Der jeweilige Stand der wissenschaftlichen Erkenntnisse ist zugrunde zu legen.

2. Es ist zu prüfen, ob der verfolgte Zweck nicht durch andere Methoden oder Verfahren erreicht werden kann.

3. Versuche an Wirbeltieren oder Kopffüßern dürfen nur durchgeführt werden, wenn die zu erwartenden Schmerzen, Leiden oder Schäden der Tiere im Hinblick auf den Versuchszweck ethisch vertretbar sind.

93 Siehe näher Lorz/Mezger, 2019: § 7 TierSchG Rdnr. 5. 
4. Schmerzen, Leiden oder Schäden dürfen den Tieren nur in dem Maße zugefügt werden, als es für den verfolgten Zweck unerlässlich ist; insbesondere dürfen sie nicht aus Gründen der Arbeits-, Zeit- oder Kostenersparnis zugefügt werden.

5. Versuche an Tieren, deren artspezifische Fähigkeit, unter den Versuchseinwirkungen zu leiden, stärker entwickelt ist, dürfen nur durchgeführt werden, soweit Tiere, deren derartige Fähigkeit weniger stark entwickelt ist, für den verfolgten Zweck nicht ausreichen." ${ }^{49}$

Tierversuche dürfen in der Zukunft also nicht mehr durchgeführt werden, wenn an ihrer Stelle einmal genau so gut Organoide z. B. für Toxizitätstests eingesetzt werden können. ${ }^{95}$

Finden die Versuche an Wirbeltieren statt, stellt § 7a Abs. 2 Nr. 3 TierSchG eine weitere Schranke auf. Versuche an Wirbeltieren dürfen nur durchgeführt werden, wenn die zu erwartenden Schmerzen, Leiden oder Schäden der Versuchstiere im Hinblick auf den Versuchszweck ethisch vertretbar sind. Es muss also eine Abwägung der Versuchsfolgen mit dem Versuchszweck stattfinden. Das Wohlergehen des Tieres wiegt umso schwerer, je geringer die Bedeutung des Versuchszwecks ist. ${ }^{96}$

\subsection{Zusammenfassung und Ausblick}

Das deutsche Recht enthält zu Organoiden keine speziellen Rechtsregeln.

Bezogen auf die Gewinnung der für die Herstellung notwendigen menschlichen Zellen und bezogen auf die Übertragung eines Organoids auf einen (anderen) Menschen bestehen jedoch in der Sache recht umfangreiche Vorschriften, die letztlich vor allem der Steuerung entsprechender Risiken für den Spender bzw. den Empfänger dienen.

Reine In-vitro-Versuche müssen nur dann von einer Ethikkommission bewertet werden, wenn dabei

1. ein Arzt einbezogen ist und

2. entweder lebendes embryonales Gewebe oder Körpermaterialien oder Daten verwendet werden, die sich einem bestimmten Menschen zuordnen lassen.

94 Näher Lorz/Mezger, 2019: § 7a TierSchG Rdnrn. $19 \mathrm{ff}$.

95 Für eine optimistische Einschätzung des Potenzials von Organoiden, Tierversuche künftig zu ersetzen, siehe das Interview mit Clevers, Kap. 2.2; für ethische Überlegungen zur Problematik siehe Schicktanz, Kap. 6.

96 Näher Hirt et al., 2016: § 7a TierSchG Rdnrn. 90 ff.; Deutscher Ethikrat, 2011: 48 f. 
Sehr wenig ist der Fragenkreis Mensch-Tier-Verbindungen durchnormiert (siehe dazu auch Schicktanz, Kap. 6). Abgesehen von den rudimentären Vorschriften des $\S 7$ ESchG, die nur selten im Fall von Organoiden einschlägig sein dürften, und abgesehen von den Vorschriften des Tierschutzgesetzes, die Tierversuche nur allgemein regeln, existieren keine einschlägigen Bestimmungen. Gerade bezüglich der Herstellung von Mensch-Tier-Organoiden ${ }^{97}$ oder auch vollständiger Mischwesen (etwa durch Implantation menschlicher Organoide in Tiere oder umgekehrt durch Einfügung tierischer Organoide in menschliche Individuen) existiert eine Reihe von Anwendungsszenarien, die den Ruf nach weiteren Vorschriften haben laut werden lassen. ${ }^{98}$ Zentral dürfte insbesondere die Forderung sein, dass entsprechende Forschung von einer Ethikkommission bewertet werden muss. ${ }^{99}$

\subsection{Literaturverzeichnis}

Akademie der Wissenschaften Schweiz (2009): Interspezies-Mischwesen. Aspekte des Tierschutzes. Unter http://www.tierethik.net/resources/609072076AltexethikSAMW.pdf [31.03.2020].

Antonov, K. (2006): Der rechtliche Rahmen der Zulässigkeit für Biobanken zu Forschungszwecken. Nomos, Baden-Baden.

Beck'scher Online-Kommentar zum BGB (01.05.2019), Bürgerliches Gesetzbuch, 50. Edition. Beck, München.

Boers, S. et al. (2016): Organoid biobanking: Identifying the ethics. In: EMBO reports 17(7): 938-941. Breithaupt, J. (2012): Rechte an Körpersubstanzen und deren Auswirkung auf die Forschung mit abgetrennten Körpersubstanzen. Nomos, Baden-Baden.

Büchner, B. (2010): Körpersubstanzen als Forschungsmaterialien. Kovac, Hamburg.

Denker, H.-W. (2017): Embryonen, Embryoide, Gastruloide. In: Rothhaar, M. et al. (Hrsg.): Der manipulierbare Embryo. Mentis, Münster, 15-47.

Deutsch, E. (1992): Das Persönichkeitsrecht des Patienten. In: Archiv für die civilistische Praxis 192(3): 161-180.

Deutscher Ethikrat (2010): Humanbiobanken für die Forschung. Eigenverlag, Berlin.

Deutscher Ethikrat (2011): Mensch-Tier-Mischwesen in der Forschung. Eigenverlag, Berlin.

Faltus, T. (2016): Stammzellenreprogrammierung. Nomos, Baden-Baden.

Fenger, H. (2018): GenTG. In: Spickhoff, A. (Hrsg.): Medizinrecht. 3. Auflage. C. H. Beck, München.

97 Dazu etwa Munsie et al., 2017: 943 f.; Hostiuc et al., 2019: 120.

98 Siehe etwa Taupitz/Weschka, 2009; Akademie der Wissenschaften Schweiz, 2009; Deutscher Ethikrat, 2011.

99 Taupitz/Weschka, 2009: 455; Deutscher Ethikrat, 2011: 120; Munsie et al., 2017: 944. 
Fink, S. (2005): Einwilligung und vertragliche Regelungen zur Entnahme von Körpersubstanzen, deren Aufbewahrung und Verwendung in Biobanken. Jur. Diss., Mannheim.

Förster, C. (2019): Kommentierung von $§ 823$ BGB. In: Beck'scher Online-Kommentar zum BGB (01.05.2019), Bürgerliches Gesetzbuch, 50. Edition. Beck, München.

v. Freier, F. (2005): Getrennte Körperteile in der Forschung zwischen leiblicher Selbstverfügung und Gemeinbesitz. In: Medizinrecht 23: 321-328.

Gerke, S. et al. (2020): Die klinische Anwendung von humanen induzierten pluripotenten Stammzellen. Springer, Berlin.

Gerke, S. (2020a): Die klinische Translation von hiPS-Zellen in Deutschland. In: Gerke et al. (Hrsg.): Die klinische Anwendung von humanen induzierten pluripotenten Stammzellen. Springer, Berlin: 243-327.

Gerke, S. et al. (2020b): Eine rechtsvergleichende Analyse der klinischen Translation von hiPS-Zellen in Deutschland und Österreich. In: Gerke, S. et al. (Hrsg.): Die klinische Anwendung von humanen induzierten pluripotenten Stammzellen. Springer, Berlin: 421-455.

Günther H.-L. (2014): Kommentierung zu § 6 und $\S 7$ ESchG. In: Günther, H.-L. et al. (Hrsg.): Embryonenschutzgesetz. 2. Auflage. Kohlhammer, Stuttgart: C. II. § 6, § 7.

Günther, H.-L. et al. (2014): Embryonenschutzgesetz. Zweite Auflage. Kohlhammer, Stuttgart.

Halasz, C. (2004): Das Recht auf bio-materielle Selbstbestimmung. Springer, Berlin.

Hirt, A. et al. (2016): Tierschutzgesetz. Vahlen, München.

Hostiuc, S. et al. (2019): The moral status of cerebral organoids. In: Regenerative Therapy 10: 118-122, Online-Publikation 27.02.2019. DOI: 10.1016/j.reth.2019.02.003.

Kopetzki C. et al. (2020): Die klinische Translation von hiPS-Zellen in Österreich. In: Gerke, S. et al. (Hrsg.): Die klinische Anwendung von humanen induzierten pluripotenten Stammzellen. Springer, Berlin: 329-420.

Krüger, M. et al. (2009): Allgemeine Vorschriften. In: Pühler, W. et al. (Hrsg.): Praxisleitfaden Gewebegesetz. Grundlagen, Anforderungen, Kommentierungen. Deutscher Ärzte-Verlag, Köln: 62-73.

Lorz, A./Mezger, E. (2019): Tierschutzgesetz. 7. Auflage. Beck, München.

Müller-Terpitz, R. (2008): Der Schutz des pränatalen Lebens. Mohr Siebeck, Tübingen.

Munsie, M. et al. (2017): Ethical issues in human organoid and gastruloid research. In: Development 144: 942-945, Online-Publikation 14.03.2017. DOI: 10.1242/dev.14011.

Nationaler Ethikrat (2004): Biobanken für die Forschung. Eigenverlag, Berlin.

Ohly, A. (2003): Die Einwilligung des Spenders von Körpersubstanzen und ihre Bedeutung für die Patentierung biotechnologischer Erfindungen. In: Festschrift für Reimar König. Heymann, Köln: 417-433.

Paul-Ehrlich-Institut (2012): Arzneimittel für neuartige Therapien. Regulatorische Anforderungen und praktische Hinweise. Unter: https://www.pei.de/SharedDocs/Downloads/DE/regulation/bera tung/innovationsbuero/broschuere-atmp.pdf?__blob=publicationFile\&v=4 [31.03.2020]. 
Prütting, H. (2018): Kommentierung zu § 12 BGB. In: Prütting, H. et al. (Hrsg.): Bürgerliches Gesetzbuch. 13. Auflage. Wolters Kluwer, Köln.

Roth, C. (2009): Eigentum an Körperteilen. Springer, Berlin.

Sass, H. M. (1989): Hirntod und Hirnleben. In: Sass, H. M. (Hrsg.): Medizin und Ethik. Reclam, Stuttgart: $160-183$.

Schaub, R. (2018): Kommentierung zu § 823 BGB. In: Prütting, H. et al. (Hrsg.): Bürgerliches Gesetzbuch. 13. Auflage. Wolters Kluwer, Köln.

Schreiber, M. (2019): Die medizinische Forschung mit abgetrennten Körpersubstanzen Minderjähriger. Lit, Münster.

Schröder, M./Taupitz, J. (1991): Menschliches Blut: Verwendbar nach Belieben des Arztes? Enke, Stuttgart.

Simon, J. et al. (2006): Biomaterialbanken. MWV, Berlin.

Söns, U. (2008): Biobanken im Spannungsfeld von Persönlichkeitsrecht und Forschungsfreiheit. Kovac, Hamburg.

Sprau, H. (2020): Kommentierung von § 823 BGB. In: Palandt, O. (Hrsg.): Bürgerliches Gesetzbuch. 79. Auflage. Beck, München.

Stein, R. (2019): Scientists Create A Device That Can Mass-Produce Human Embryoids. In: NPR, Online Publikation 11.09.2019. Unter: https://www.npr.org/sections/health-shots/2019/09/11/75707299 6/scientists-create-a-device-that-can-mass-produce-synthetic-human-embryos [31.03.2020].

Taupitz, J. (1991): Wem gebührt der Schatz im menschlichen Körper? In: Archiv für die civilistische Praxis 191(3): 201-246.

Taupitz, J. (2001): Der rechtliche Rahmen des Klonens zu therapeutischen Zwecken. In: Neue Juristische Wochenschrift (47): 3433-3440.

Taupitz, J. (2009): Bindungswirkung von Standards im Gesundheitswesen. In: Möllers, T. (Hrsg.): Geltung und Faktizität von Standards. Nomos, Baden-Baden: 63-106.

Taupitz, J. (2014): Kommentierung zu § 8 ESchG. In: Günther, H.-L. et al. (Hrsg.): Embryonenschutzgesetz. Zweite Auflage. Kohlhammer, Stuttgart: C. II. § 8.

Taupitz, J. (2017): Der Verkauf von Restblut an die Medizinprodukteindustrie. In: Medizinrecht, 35: 353-361, Online-Publikation 15.05.2017. DOI: 10.1007/s00350-017-4595-z.

Taupitz, J./Schreiber, M. (2016): Biobanken - zwischen Forschungs- und Spenderinteressen. In: Bundesgesundheitsblatt (59): 304-310.

Taupitz, J./Weschka, M. (2009): CHIMBRIDS. Chimeras and Hybrids in comparativ European and international research. Springer, Berlin.

Wernscheid, V. (2012): Tissue Engineering. Rechtliche Grenzen und Voraussetzungen. Universitätsverlag, Göttingen.

Wicklein, M. (2007): Biobanken zwischen Wissenschaftsfreiheit, Eigentumsrecht und Persönlichkeitsschutz. Tectum, Marburg. 
Zentrale Ethikkommission bei der Bundesärztekammer (2003): Die (Weiter-)Verwendung von menschlichen Körpermaterialien für Zwecke medizinischer Forschung. Unter: https://www.ze ntrale-ethikkommission.de/fileadmin/user_upload/downloads/pdf-Ordner/Zeko/Koerpermat-1.p df [31.03.2020]. 